\title{
Dynamique des Adventices dans la Culture de Canne À Sucre : Cas de l'Unité Agricole Intégrée de Zuénoula (Centre-Ouest de la Côte d'Ivoire)
}

\author{
Karidia Traoré, Maître de Conférences \\ Karnon Ouattara, Doctorant \\ Moussa Sylla, Assistant \\ Siendou Coulibaly, Maître-assistant
}

Université Jean Lorougnon Guédé, UFR Agroforesterie, Côte d'Ivoire

Doi:10.19044/esj.2019.v15n21p361 URL:http://dx.doi.org/10.19044/esj.2019.v15n21p361

Résumé

L'étude a porté sur la dynamique des adventices de la canne à sucre à l'Unité Agricole intégrée de Zuénoula. Elle avait pour objectif de contribuer à la gestion de l'enherbement dans cette unité agricole. À cet effet, des relevés floristiques ont été réalisés dans 3 classes d'âges de culture de la canne à sucre par la méthode des transects. Ainsi, 133 espèces d'adventices réparties en 36 familles et 92 genres ont été recensées. Les Dicotylédones sont mieux représentées avec 96 contre 37 espèces de Monocotylédones. Dans l'ensemble des classes d'âges, les familles les plus riches en espèces étaient les Poaceae, les Fabaceae, les Euphorbiaceae et les Asteraceae. L'indice de diversité spécifique a montré que le nombre d'espèces adventices augmente avec l'âge de la culture. Ces adventices sont floristiquement homogènes. Certaines espèces telles que Cynodon dactylon, Cyperus rotundus, Dioscorea bulbifera et Ipomoea aquatica ont été plus agressives dans des cultures de 0 à 3 mois. Les plus agressives au niveau des cultures de 4 à 6 mois ont été Croton lobatus, Cyperus rotundus, Rottboellia cochinchinensis, Ipomoea involucrata et Momordica charantia. Les parcelles cannières d'au moins 7 mois sont particulièrement agressées par Cynodon dactylon, Ipomoea aquatica, Momordica charantia et Porophyllum ruderale. Les résultats de la présente étude mettent alors en évidence la chronologie d'apparition des adventices dans le cycle cultural de la canne à sucre. Cela devrait permettre la prise de décision optimale pour le désherbage afin d'améliorer la gestion de l'enherbement.

Mots-clés : Dynamique, mauvaise herbe, classe d'âge, canne à sucre 


\title{
Dynamic Weed in Sugar Cane Cultivation: Case of Integrated Agricultural Unit of Zuénoula (Central West of Côte d'Ivoire)
}

\author{
Karidia Traoré, Maître de Conférences \\ Karnon Ouattara, Doctorant \\ Moussa Sylla, Assistant \\ Siendou Coulibaly, Maître-assistant
}

Université Jean Lorougnon Guédé, UFR Agroforesterie, Côte d'Ivoire

Abstract

This paper focuses on the dynamics of sugarcane weeds at the Integrated Agricultural Unit of Zuénoula. Its purpose was to contribute to the management of grass cover in this agricultural unit. For this purpose, floristic surveys were carried out in three (3) age classes of sugarcane cultivation using the transect method. Thus, 133 species of weeds divided into 36 families and 92 genera were recorded. Dicotyledons are best represented with 96 against 37 species of Monocotyledons. In all age groups, the richest families' species were Poaceae, Fabaceae, Euphorbiaceae, and Asteraceae. The species diversity index has shown that the number of weed species increases with the age of the crop. These weeds are floristically homogeneous. Some species such as Cynodon dactylon, Cyperus rotundus, Dioscorea bulbifera and Ipomoea aquatica have been more aggressive in 0-3 month old crops. The most aggressive at 4 to 6 month old crops were Croton lobatus, Cyperus rotundus, Rottboellia cochinchinensis, Ipomoea involucrate, and Momordica charantia. The cane plots of at least 7 months are particularly attacked by Cynodon dactylon, Ipomoea aquatica, Momordica charantia and Porophyllum ruderale. The results of this study highlight the chronology of weed occurrence in the sugarcane crop cycle. This should enable optimal decision-making for weed control to improve weed management.

Keywords: Dynamics, Weeds, Age Class, Sugar cane

\section{Introduction}

Originaire de l'archipel de la Nouvelle-Guinée, la canne à sucre aurait été répandue par l'homme dans toutes les îles du Pacifique et dans l'océan Indien jusqu'en Malaisie, dans la péninsule indochinoise (Van Dillewijn, 
1960). En Afrique, c'est au XVI ${ }^{\mathrm{e}}$ siècle que la canne à sucre fut implantée, puis longtemps exploitée de manière empirique (Péné, 1999).

En Côte d'Ivoire, c'est à partir de l'année 1964 que la culture de canne à sucre s'installe de manière expérimentale (Péné, 1999). De nos jours le pays est classé $53^{\text {ème }}$ mondial et $16^{\text {ème }}$ africain. Sa production s'élève à environ 330 $000 \mathrm{t}$ de sucre, soit $50 \%$ de la consommation de l'espace UEMOA (Union Économique et Monétaire Ouest Africaine). Le marché de l'UEMOA offre, à moyen terme, de bonnes perspectives de développement pour les entreprises sucrières ivoiriennes (Kouamé et al., 2009 ; Sangaré et al., 2009).

Il est cependant important de noter qu'à l'instar des autres cultures, la monoculture industrielle cannière reste fortement influencée par des facteurs aussi bien abiotiques que biotiques. Parmi les facteurs biotiques, la culture de la canne à sucre est très sensible à l'enherbement (Lebreton, 2010). En effet, un temps d'entretien de 4 mois est nécessaire pour que la canne ne subisse pas une perte de rendement mesurable suite à la compétition induite par les mauvaises herbes (Raji, 1995). En Côte d'Ivoire, les pertes de rendement dues aux adventices sont estimées entre 400 et $500 \mathrm{~kg}$ de canne par hectare et par jour de retard de désherbage pour une variété au potentiel de 130 tonnes de canne à l'hectare (Marion \& Marnotte, 1991). Raji (1995) a montré que la présence des mauvaises herbes durant tout le cycle de la canne pourrait réduire le rendement de 85 à $89 \%$. Le désherbage des parcelles de culture est donc une préoccupation majeure pour l'agriculteur cannier (Lebreton, 2010).

En Côte d'Ivoire, pour contribuer à la gestion durable des mauvaises herbes en culture cannière, plusieurs études ont traité de leur systématique, leur biologie et leur écologie (Boraud \& Aman, 1998 ; Boraud, 2000 ; Traoré et al., 2014). Cependant, les travaux relatifs à l'étude de la dynamique des adventices dans la culture de la canne à sucre sont plutôt peu nombreux ou mal diffusés. Pourtant, dans une culture intensive comme celle de la canne à sucre où l'on retrouve, sur un même espace, la même espèce à différents stades de son développement, il est important de prendre en compte, dans les programmes de gestion, la dynamique des adventices rencontrées. C'est dans ce contexte qu'intervient la présente étude. L'hypothèse à vérifier est la suivante : la flore adventice subit une évolution au cours du cycle cultural de la canne à sucre. À l'égard de cette hypothèse, cette étude a pour objectif général d'améliorer les itinéraires techniques de désherbage de la canne à sucre et l'époque d'intervention selon son cycle de développement. De façon spécifique, il s'agira de :

$\checkmark$ caractériser la flore adventice de la canne à sucre ;

connaître la chronologie d'apparition des adventices majeures durant le développement de la canne à sucre. 


\section{Matériel et méthodes \\ Matériel}

Le matériel biologique est constitué de l'ensemble des adventices inventoriées dans les cultures cannières de l'Unité Agricole Intégrée de Zuénoula (UAI). Il comprend les adventices herbacées et lianescentes.

Le matériel technique comprend essentiellement une carte parcellaire mise à notre disposition par le Service des Études Agronomiques (SEA) comme support d'échantillonnage.

\section{Description de la zone d'étude}

L'étude a été conduite dans la localité de Zuénoula (Centre-ouest de la Côte d'Ivoire à $400 \mathrm{~km}$ d'Abidjan), plus précisément à l'UAI de la société SUCRIVOIRE. Ce domaine agro-industriel est situé à $25 \mathrm{~km}$ au Nord de ladite localité, et il est à cheval entre la région de la Marahoué et du Haut-Sassandra (Anonyme, 2010) (Figure 1). Les coordonnées géographiques de l'UAI sont : de $7^{\circ} 37^{\prime}$ à $7^{\circ} 39^{\prime}$ de latitude Nord, et de $6^{\circ} 11^{\prime}$ à $6^{\circ} 14^{\prime}$ de longitude Ouest, avec une altitude de 209 m (Péné \& Assa, 2003). Le régime climatique est celui du domaine guinéen caractérisé par 2 grandes saisons : une saison sèche de 3 mois (novembre-janvier) et une saison pluvieuse de 9 mois (févrieroctobre). La pluviométrie moyenne annuelle est de $1216 \mathrm{~mm}$ d'eau et la température moyenne est de $26,74{ }^{\circ} \mathrm{C}$ (Anonyme, 2017). La localité de Zuénoula appartient au secteur mésophile. La végétation initiale est constituée de la forêt dense humide semi-décidue (Pamentier, 2007). On y rencontre des sols ferralitiques fortement à moyennement désaturés, avec une bonne aptitude agricole (Péné, 1999). Le principal cours d'eau, la Marahoué ou Bandama rouge, forme un chevelu relativement dense qui a des répercussions sur la répartition des blocs sucriers (Boraud, 2000). 


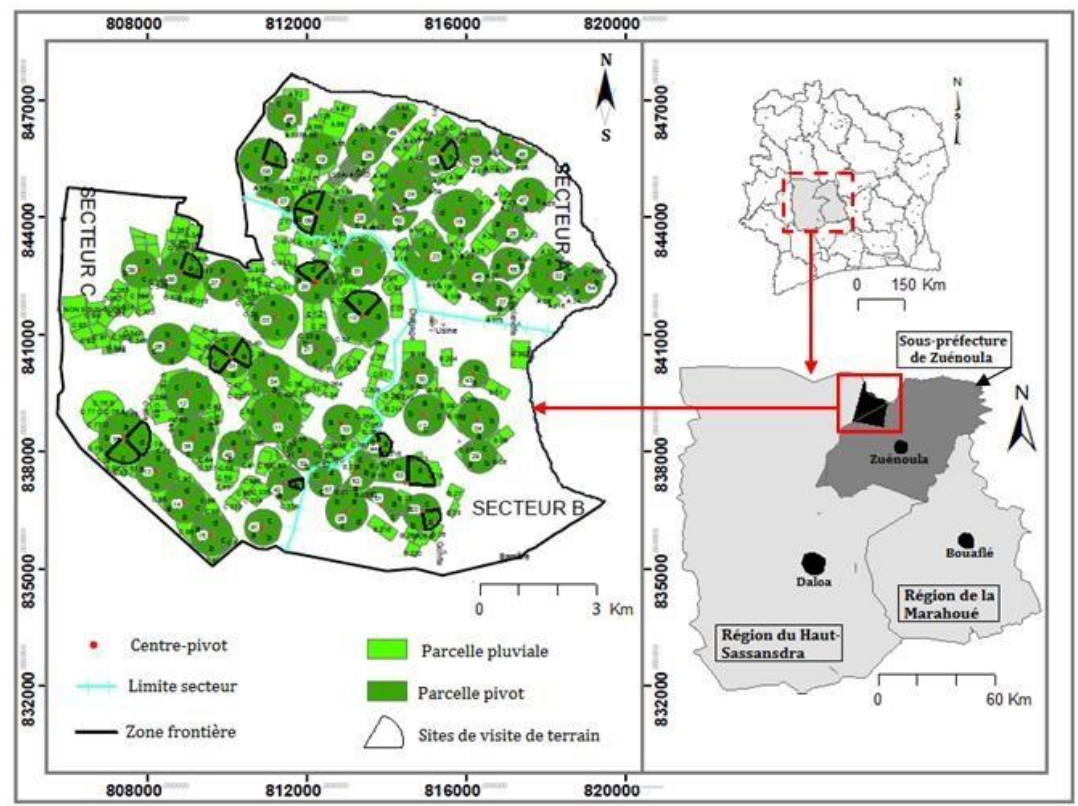

Figure 1. Carte de localisation de la zone d'étude

\section{Méthodes}

\section{Relevés floristiques}

Les relevés floristiques ont été réalisés selon la méthode des transects (Meddour, 2011) sur 15 parcelles de canne à sucre, dont 5 de 0 à 3 mois de culture (dénommées $\mathrm{C} 1$ ) ; 5 de 4 à 6 mois $(\mathrm{C} 2)$ et 5 parcelles de 7 mois et plus (C3). Dans chaque parcelle, 16 interlignes de $250 \mathrm{~m}$ de longueur ont été parcourues sur une largueur de $1,5 \mathrm{~m}$, soit $375 \mathrm{~m}^{2}$. Toutes les espèces rencontrées ont été identifiées et notées en présence-absence sans tenir compte du nombre d'individus. Les identifications ont été faites à l'aide du manuel de référence Adventices tropicales de Merlier et Montegut (1982) et des spécialistes en la matière.

\section{Évaluation de la diversité floristique des adventices}

La diversité de la flore adventice a été caractérisée par classe d'âges et pour l'ensemble des classes d'âges confondues, à travers trois indices :

3. l'indice de diversité générique (Ig), qui est le rapport entre le nombre de genres et celui de familles ;

4. l'indice de diversité spécifique (Is), qui est le rapport entre le nombre d'espèces et celui de genres ;

5. le taux des classes botaniques (Monocotylédones et Dicotylédones), qui est le rapport entre le nombre d'espèces de la classe et le nombre total d'espèces recensées, multiplié par 100. 


\section{Évaluation de la similitude de la flore adventice}

Le coefficient de similitude a permis d'analyser l'homogénéité des adventices des différents stades culturaux. Il a été calculé en opposant deux à deux les listes floristiques des différentes classes d'âges, selon la formule de Sørensen (1948) :

$$
\mathrm{Cs}=\left(\mathbf{2} \mathrm{c}^{* 100}\right) /(\mathbf{a}+\mathbf{b})
$$

Où $\mathrm{a}$ et $\mathrm{b}$ représentent le nombre d'espèces recensées respectivement dans les deux classes d'âges à comparer et $\mathrm{c}$ le nombre d'espèces communes aux deux classes. En théorie, Cs varie entre 0 et $100 \%$, mais dans la pratique, ces valeurs limites ne sont presque jamais atteintes. Lorsque Cs est supérieur ou égal à 50\%, cela signifie que les deux listes comparées sont très proches l'une de l'autre au point d'être assimilées à des milieux identiques. En d'autres termes, cela indique que les deux classes d'âges concernées sont floristiquement homogènes. Par contre, lorsque les deux listes ont des compositions floristiques différentes, Cs est inférieur à $50 \%$ (Aman et al., 2004).

\section{Fréquence relative}

La fréquence relative ou la représentativité d'une adventice permet d'avoir une idée sur la répartition de l'espèce sur un espace bien précis. La fréquence relative (Fr) d'une espèce végétale donnée, exprimée en pourcentage, se définit comme le rapport de sa fréquence absolue $(\mathrm{Fa})$ ou nombre de relevés où elle est présente au nombre total $(\mathrm{N})$ de relevés effectués sur un site donné. Elle se traduit par l'expression suivante :

$$
\mathbf{F r}=(\mathbf{F a} / \mathbf{N}) \times 100
$$

La fréquence relative ou la représentativité a été calculée pour chaque espèce agressive de chaque classe d'âge.

\section{Contribution spécifique}

La contribution spécifique C.S.F.(e) due à la fréquence spécifique d'une espèce (e) renseigne sur la participation de celle-ci à la végétation. C'est l'expression de l'agressivité ou de la productivité de l'espèce sur le terrain (Aman, 1978). Elle s'obtient par le rapport de la fréquence spécifique de l'espèce F.S.(e) à la somme des fréquences spécifiques de toutes les espèces recensées dans l'unité d'échantillonnage ( ${ }^{\sum_{\mathrm{i}}^{\mathrm{n}} \mathrm{F} . \mathrm{S} \text {. (e) }}$ ), multiplié par 100 :

$$
\text { C.S.F }(e)=\frac{\text { F.S. (e) }}{\sum_{\mathrm{i}}^{\mathrm{n}} \text { F.S. (e) }} \times 100
$$

Cette contribution spécifique est interprétée comme suit (Daget et Poissonet, 1971) : 
$\checkmark 1 \leq$ C.S.F. $\leq 4$ : espèces peu agressives ou adventices majeures potentielles ;

$\checkmark$ C.S.F. $\geq 4$ : espèces très agressives ou adventices majeures.

La contribution spécifique due à la fréquence spécifique de l'espèce a été calculée dans chaque classe d'âge.

\section{Résultats}

\section{Richesse floristique}

Les relevés floristiques ont permis d'identifier 133 espèces d'adventices appartenant à 36 familles botaniques et 92 genres. La classe des Dicotylédones est mieux représentée $(72,18 \%$ des espèces) que celle des Monocotylédones $(27,82 \%)$ (Figure 2). Les familles les plus dominantes sont les Poaceae (25 espèces), les Fabaceae (12 espèces), les Euphorbiaceae (11 espèces) et les Asteraceae (10 espèces). Ces quatre familles renferment environ $43 \%$ de la flore adventice (Figure 3). Les espèces lianescentes représentent 17,29 p.c. des adventices, soit 23 espèces répertoriées, principalement dans les familles des Convolvulaceae, Dioscoreaceae et Fabaceae.

Dans les cultures de 0 à 3 mois, 82 espèces d'adventices ont été inventoriées, dont 60 Dicotylédones $(73,17 \%)$ et 22 Monocotylédones (26,83\%). Dans les cultures de 4 à 6 mois, 94 espèces d'adventices ont été identifiées dont $71,28 \%$ de Dicotylédones (67 espèces) et $28,72 \%$ de Monocotylédones (27 espèces). Quant aux cultures de 7 mois et plus, elles sont occupées par 87 espèces d'adventices dont 71,26\% de Dicotylédones (62 espèces) et $28,73 \%$ de Monocotylédones (25 espèces).

Les indices de diversité générique (Ig) et spécifique (Is) des différentes classes d'âges de la culture cannière sont consignés dans le tableau I. L'Ig a été de 2,55 pour l'ensemble des cultures. Il a varié de 1,71 dans les cultures de 7 mois et plus à 2,43 dans celles de 4 à 6 mois. Quant à l'Is, il a été de 1,43 dans l'ensemble des cultures. Il a augmenté avec l'âge des cultures, de 1,28 dans les cultures de $0-3$ mois à 1,33 dans celles de 7 mois et plus.

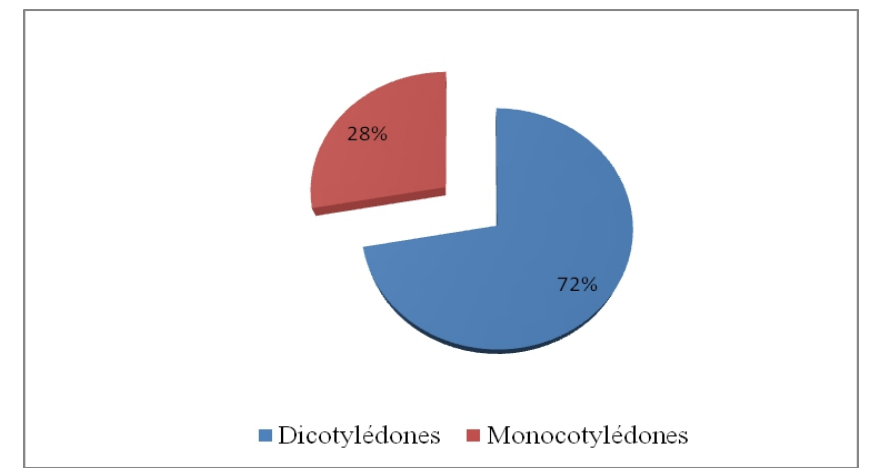

Figure 2. Diagramme représentatif des Dicotylédones et des Monocotylédones 


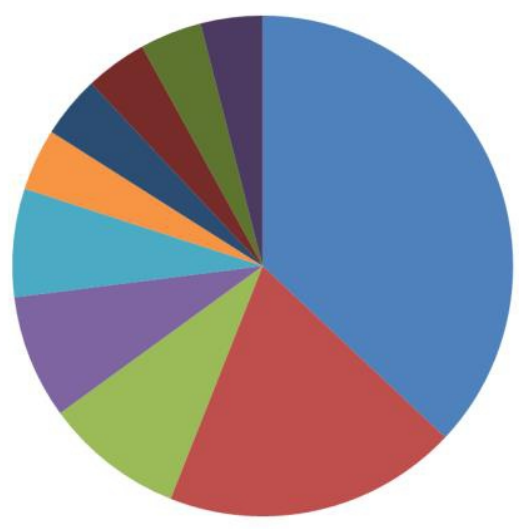

Figure 3. Spectre des familles dominantes en culture cannière

Tableau I. Indices de diversité générique et spécifique dans les classes d’âges

\begin{tabular}{|ccccc|}
\hline & \multicolumn{4}{c|}{ Classes d'âge } \\
\cline { 2 - 5 } Indice & $\mathbf{C 1}$ & $\mathbf{C 2}$ & $\mathbf{C 3}$ & Total \\
\hline Ig & 2,17 & 2,43 & 1,71 & 2,55 \\
Is & 1,28 & 1,3 & 1,33 & 1,43 \\
\hline
\end{tabular}

C1 : 0-3 mois ; C2 : 4-6 mois ; C3 : 7 mois et plus ; Ig : indice de diversité générique ; Is : indice de diversité spécifique.

\section{Similitude de la flore adventice}

Les valeurs des coefficients de similitudes sont comprises entre 67 et $69 \%$ (Tableau II). Elles sont nettement supérieures à 50\%, ce qui signifie que les adventices des différents âges de cultures sont identiques. On dirait, en d'autres termes, que les parcelles des différents cycles culturaux de la canne à sucre sont floristiquement homogènes.

Tableau II. Coefficients de similitude entre les listes floristiques d'adventices des cultures cannières de différents âges

\begin{tabular}{|lc|}
\hline $\begin{array}{c}\text { Couple de classes } \\
\text { d'âges }\end{array}$ & Cs $(\%)$ \\
\hline C1 et C2 & 67,04 \\
C1 et C3 & 66,66 \\
C2 et C3 & 69,23 \\
\hline
\end{tabular}

C1 : 0-3 mois ; C2 : 4-6 mois ; C3 : 7 mois et plus ; Cs : Coefficient de similitude 


\section{Nuisibilité des adventices}

La Contribution Spécifique due à la Fréquence Spécifique de chacune des 133 espèces d'adventices, a permis de dresser une classification de toutes ces espèces d'adventices durant le cycle cultural de la canne à sucre. Ainsi, les adventices les plus agressives sont présentées ici (C.S.F. $\geq 4$ ) dans chaque classe d'âge.

Le tableau III présente les espèces d'adventices les plus agressives rencontrées dans les cultures de 0 à 3 mois. Les adventices inventoriées dans cette classe d'âge sont aux nombres de 19, dont 15 herbacées et 4 lianescentes. Ceux sont entre autres: Cynodon dactylon et Cyperus rotundus pour les herbacées et Momordica charantia et Dioscorea bulbifera pour les lianescentes.

Dans les parcelles de 4 à 6 mois de culture, les espèces d'adventices les plus productives sont consignées dans le tableau IV. Il a été identifié dans cette classe d'âge 19 espèces d'adventices (14 herbacées et 5 lianescentes). Les plus nuisibles sont Croton lobatus et Cyperus rotundus pour les herbacées et Momordica charantia et Dioscorea bulbifera pour les lianescentes.

Les adventices majeures inventoriées dans les parcelles de 7 mois et plus sont au nombre de 26 espèces dont 18 herbacées et 8 lianescentes (Tableau V). Les plus productives sont Cynondon dactylon et Porophyllum ruderale pour les herbacées et Ipomoea aquatica et Ipomoea involucrata pour les lianescentes.

Tableau III. Liste des adventices majeures des cultures cannières de 0 à 3

Mois

\begin{tabular}{|c|c|c|c|}
\hline $\begin{array}{c}\text { Type } \\
\text { d'adventice }\end{array}$ & Espèces & C.S.F. & $\begin{array}{c}\text { Représentativité } \\
(\%)\end{array}$ \\
\hline \multirow{10}{*}{ Herbacées } & Cynodon dactylon (L.) Persoon & 12,03 & 80 \\
\hline & Cyperus rotundus $\mathrm{L}$. & 12,03 & 60 \\
\hline & Amaranthus viridis $\mathrm{L}$. & 7,52 & 20 \\
\hline & Rottboellia cochinchinensis (Loureiro) Clayton & 6,37 & 80 \\
\hline & Physalis angulata $\mathrm{L}$. & 6,3 & 40 \\
\hline & Phyllanthus amarus Schumacher \& Thonning & 5,91 & 40 \\
\hline & Cyperus difformis $\mathrm{L}$. & 5,52 & 20 \\
\hline & $\begin{array}{l}\text { Launea cornuta (Hochstetter ex Oliver \& Hiem) } \\
\text { Jeffrey }\end{array}$ & 5,26 & 20 \\
\hline & Commelina benghalensis L. & 5,18 & 100 \\
\hline & Erigeron floribundus (H. B. \& K.) Sch. Bip. & 5,17 & 20 \\
\hline
\end{tabular}




\begin{tabular}{|l|l|c|c|}
\hline \multirow{5}{*}{$\begin{array}{l}\text { Chromolaena odorata (L.) R. M. King \& H. } \\
\text { Robinson }\end{array}$} & 4,78 & 40 \\
\cline { 2 - 4 } & Kyllinga squamulata Thonning ex Vahl & 4,69 & 20 \\
\cline { 2 - 4 } & Panicum maximum Jacquin & 4,38 & 20 \\
\cline { 2 - 4 } & Sida acuta Burman F. & 4,14 & 20 \\
\cline { 2 - 4 } & Euphorbia hirta L. & 4,11 & 20 \\
\hline & Momordica charantia & 5,12 & 20 \\
\cline { 2 - 4 } & Dioscorea bulbifera L. & 4,83 & 20 \\
\cline { 2 - 4 } & Ipomoea involucrata P.Beauv. & 4,33 & 20 \\
\cline { 2 - 4 } & Centrosema pubescens Benth. & 4,14 & 20 \\
\hline
\end{tabular}

C.S.F : Contribution Spécifique due à la Fréquence Spécifique de l'espèce

Tableau IV. Liste des adventices majeures des cultures cannières de 4 à 6 mois

\begin{tabular}{|c|c|c|c|}
\hline $\begin{array}{c}\text { Type } \\
\text { d'adventice }\end{array}$ & Espèces & C.S.F. & $\begin{array}{c}\text { Représentativité } \\
(\%)\end{array}$ \\
\hline \multirow{14}{*}{ Herbacées } & Croton lobatus L. & 5,19 & 20 \\
\hline & Cyperus rotundus $\mathrm{L}$. & 5,19 & 40 \\
\hline & Rottboellia cochinchinensis (Loureiro) Clayton & 5,19 & 40 \\
\hline & Commelina benghalensis L. & 4,86 & 60 \\
\hline & Cynodon dactylon (L.) Persoon & 4,18 & 20 \\
\hline & Panicum maximum Jacquin & 4,02 & 20 \\
\hline & Euphorbia heterophylla $\mathrm{L}$. & 4,02 & 20 \\
\hline & Hymenocardia ulmoides Oliv & 4,02 & 20 \\
\hline & Laportea aestuens (L.) Chew & 4,02 & 20 \\
\hline & Porophyllum ruderale (Jacq.) & 4,02 & 20 \\
\hline & Portulaca oleracea Linn. & 4,02 & 20 \\
\hline & Solanum nigrum $\mathrm{L}$. & 4,02 & 20 \\
\hline & Spermacoce ruellia DC. & 4,02 & 20 \\
\hline & Tribulus terrestris Linnaeus & 4,02 & 20 \\
\hline
\end{tabular}




\begin{tabular}{|l|l|c|c|}
\hline \multirow{5}{*}{ Lianescentes } & Momordica charantia L. & 5,19 & 20 \\
\cline { 2 - 4 } & Dioscorea bulbifera L. & 4,87 & 60 \\
\cline { 2 - 4 } & Ipomoea involucrata P. Beauv. & 4,86 & 40 \\
\cline { 2 - 4 } & Passiflora foetida L. & 4,55 & 20 \\
\cline { 2 - 4 } & Centrosema pubescens Benth. & 4,02 & 20 \\
\hline
\end{tabular}

C.S.F : Contribution Spécifique due à la Fréquence Spécifique de l'espèce

Tableau V. Liste des adventices majeures des cultures cannières de 7 mois et plus

\begin{tabular}{|c|c|c|c|}
\hline $\begin{array}{c}\text { Type } \\
\text { d'adventice }\end{array}$ & Espèces & C.S.F. & $\begin{array}{c}\text { Représentativité } \\
(\%)\end{array}$ \\
\hline \multirow{18}{*}{ Herbacées } & Cynodon dactylon (L.) Persoon & 6,47 & 40 \\
\hline & Porophyllum ruderale (Jacq.) & 5,47 & 40 \\
\hline & Eleusine indica (L.) Gaertner & 5,44 & 20 \\
\hline & Hymenocardia ulmoides Oliv. & 5,44 & 20 \\
\hline & Digitaria horizontalis Willdenow & 5,1 & 20 \\
\hline & Amaranthus viridis $\mathrm{L}$. & 4,98 & 20 \\
\hline & Commelina benghalensis $\mathrm{L}$. & 4,98 & 40 \\
\hline & Cyperus rotundus $\mathrm{L}$. & 4,98 & 40 \\
\hline & Panicum maximum Jacquin & 4,98 & 20 \\
\hline & Cyperus difformis $\mathrm{L}$. & 4,48 & 20 \\
\hline & Brachiaria lata (Schumacher) C.E. Hubbard & 4,42 & 20 \\
\hline & Dactyloctenium aegyptium (L.) P. Beauv. & 4,42 & 20 \\
\hline & $\begin{array}{l}\text { Chromolaena odorata (L.) R. M. King \& H. } \\
\text { Robinson }\end{array}$ & 4,27 & 40 \\
\hline & Rottboellia cochinchinensis (Loureiro) Clayton & 4,27 & 20 \\
\hline & Phyllanthus amarus Schumacher \& Thonning & 4,23 & 20 \\
\hline & Ricinus communis $\mathrm{L}$. & 4,23 & 20 \\
\hline & Amaranthus spinosus $\mathrm{L}$. & 4,08 & 20 \\
\hline & Physalis angulata $\mathrm{L}$. & 4,08 & 20 \\
\hline \multirow{2}{*}{ Lianescentes } & Ipomoea aquatica Forskaal & 6,97 & 40 \\
\hline & Ipomoea involucrata $\mathrm{P}$. Beauv. & 5,97 & 20 \\
\hline
\end{tabular}




\begin{tabular}{|l|l|c|c|}
\hline Momordica charantia L. & 5,47 & 40 \\
\cline { 2 - 4 } & Passiflora foetida L. & 5,1 & 20 \\
\cline { 2 - 4 } & Dioscorea bulbifera L. & 4,48 & 60 \\
\cline { 2 - 4 } & Periploca nigrescens Afzel. & 4,42 & 20 \\
\cline { 2 - 4 } & Centrosema pubescens Benth. & 4,22 & 20 \\
\cline { 2 - 4 } & Dioscorea praehensilis Benth. & 4,22 & 20 \\
\hline
\end{tabular}

C.S.F : Contribution Spécifique due à la Fréquence Spécifique de l'espèce

\section{Discussion}

La flore adventice de la canne à sucre cultivée à l'U.A.I de Zuénoula est très diversifiée. Elle est composée de 133 espèces réparties en 36 familles botaniques et 92 genres. Pour le même site, Boraud (2000) a dénombré 142 espèces adventices appartenant à 43 familles et 108 genres et Traoré et al. (2014) ont inventorié 129 espèces regroupées en 30 familles et 83 genres. On peut donc remarquer que la flore adventice n'a pas significativement évolué dans le temps. Cela peut s'expliquer par une bonne maîtrise des pratiques culturales visant à lutter contre l'enherbement. Cependant, sur le plan qualitatif, une recrudescence des espèces lianescentes a été observée. En effet, 23 espèces lianescentes ont été répertoriées sur l'ensemble du complexe sucrier, contre 12 espèces selon Boraud (2000). Des résultats similaires ont été rapportés par Lebreton (2010) qui a inventorié 25 espèces lianescentes. Cette augmentation du nombre d'adventices lianescentes ces dernières années peut s'expliquer par l'adoption de techniques de coupe innovantes qui permettent d'avoir une grande quantité de paille sur les parcelles récoltées. Ce paillis, selon Hatil et al. (2008), limite la pousse des espèces herbacées mais favorise les espèces lianescentes.

Les familles botaniques les plus diversifiées ont été les Poaceae, les Fabaceae, les Euphorbiaceae et les Asteraceae. Ces 4 familles font partie des 10 familles considérées par Akobundu (1987) et par Traoré et al. (2014) comme celles des mauvaises herbes mondiales. Les Poaceae, les Asteraceae et les Fabaceae sont dominantes dans l'ensemble des trois classes d'âge de la culture cannière. L'importance des Poaceae et des Fabaceae s'explique par une bonne capacité de propagation de leurs semences et par leur adaptation aux différents milieux de culture (Fenni, 2003 ; Ipou Ipou et al., 2011).

L'indice de diversité spécifique a montré que plus la culture de la canne à sucre avance en âge, plus le nombre d'espèces d'adventices augmente, ce qui bonifie la diversité spécifique. Cela pourrait s'expliquer par la rémanence des herbicides. En effet, la rémanence, qui est la durée pendant laquelle l'effet escompté d'une matière active s'exprime, disparaît au fur et à mesure que la 
culture prend de l'âge. Ainsi, les semences d'adventices inhibées par les herbicides peuvent germer progressivement au-delà de la période de rémanence.

Les coefficients de similitudes ont été tous supérieurs à 50\%, ce qui signifie que les espèces d'adventices des différents cycles culturaux de la canne à sucre sont principalement les mêmes. Ces résultats corroborent ceux de Boraud et Aman (1998) qui estiment que les parcelles de canne à sucre ont probablement un cortège floristique commun de base relativement important. De plus, Boraud (2000) et Traoré et al. (2014) ont montré que plus de 60\% des espèces à différents cycles de la canne à sucre sont identiques. Cela montre une certaine monotonie dans la diversité floristique des adventices en Afrique tropicale (Marnotte, 2000). Nous pouvons aussi expliquer cette similarité des adventices de la canne à sucre de différents âges par l'usage intensif et régulier d'herbicides, lesquels réduisent les contingents floristiques par le phénomène de sélection des mauvaises herbes, à tel point que la flore est peu diversifiée (Mangara et al., 2010).

Parmi les adventices majeures, certaines espèces ont été communes aux trois classes d'âge. Elles se présentent donc comme les espèces les plus coriaces. Il s'agit de: Centrosema pubescens, Cynodon dactylon, Cyperus rotundus, Dioscorea bulbifera, Ipomoea involucrata, Momordica charantia, Panicum maximum et Rottboellia cochinchinensis. Ces adventices font partie des espèces majeures identifiées par Lebreton (2010). Elles sont difficiles à éradiquer et livrent une forte concurrence à la culture depuis les premiers mois de son installation jusqu'à la récolte. Marion et Marnotte (1991) ont montré également que ces espèces causent des dommages importants dans les cultures de canne à sucre et induisent des pertes importantes de production.

\section{Conclusion}

Les relevés floristiques effectués en culture cannière à l'Unité Agricole Intégrée de Zuénoula ont permis d'inventorier 133 espèces d'adventices appartenant à 36 familles et à 92 genres. Les Dicotylédones sont mieux représentées avec 93 contre 39 espèces de Monocotylédones. Les familles les plus diversifiées ont été les Poaceae, les Fabaceae, les Euphorbiaceae et les Asteraceae avec respectivement 25, 12, 11 et 10 espèces. Une recrudescence des espèces lianescentes a été notée. L'indice de diversité spécifique a montré que le nombre d'espèces adventices augmente avec l'âge de la culture. Toutefois, plus de $66 \%$ des espèces adventices ont été présentes à tous les stades de développement de la canne à sucre. Les adventices les plus agressives dans toutes les classes d'âges de la culture ont été Centrosema pubescens, Cynodon dactylon, Cyperus rotundus, Dioscorea bulbifera, Ipomoea involucrata, Momordica charantia, Panicum maximum et Rottboellia cochinchinensis. 
À partir de ces résultats, nous pouvons avancer que la flore adventice subit une évolution au cours du cycle cultural de la canne à sucre. Ces informations doivent permettre d'améliorer la gestion de l'enherbement de la canne à sucre et de raisonner les industriels quant à la prise de décision pour une lutte efficace contre les adventices.

\section{References:}

1. Akobundu, I.O. (1987). Weed Science in the tropics. Principles and practices. Wiley ed., $522 \mathrm{p}$.

2. Aman, K.G. (1978). Flore et végétation des adventices dans l'hévéaculture en basse Côte d'Ivoire (Station expérimentale de I.R.C.A). Etude écologique: Dynamique et structure. Thèse de Doctorat $3^{\text {ème }}$ cycle, Université de Cocody, Côte d'Ivoire, $194 \mathrm{p}$.

3. Aman, K.G., Ipou, I.J. \& Touré, Y. (2004). La flore des adventices des cultures cotonnières de la région du Worodougou, au Nord-ouest de la Côte d'Ivoire. Agrononomie Africaine, 16 (1) : 1-14.

4. Anonyme (2010). Spécialiste du sucre/ Sugar specialist. Livret Sucrivoire, $28 \mathrm{p}$.

5. Anonyme (2017). Conseil régional de la Marahoué, www.regionmarahoue.ci (consulté le 20/04/2018).

6. Bertrand, M. \& Doré, T. (2008). Comment intégrer la maîtrise de la flore adventice dans le cadre général d'un système de production intégrée ? Innovation Agronomique 3, I.N.R.A, UMR d'Agronomie, Paris, France, pp 1-13.

7. Boraud, N.K.M. \& Aman, K.G. (1998). Les principales adventices de la canne à sucre en Côte d'Ivoire : similitudes et différences. XVII ème conf. Sur la lutte contre les mauvaises herbes, Dijon, France, pp 11731179.

8. Boraud, N.K.M. (2000). Etude floristique et phytoécologique des adventices des complexes sucriers de Côte d'Ivoire : Ferké I, Ferké II, Borotou-koro et Zuénoula. Thèse de doctorat $3^{\mathrm{ème}}$ cycle, Université de Cocody, Abidjan, Côte d'Ivoire, 186 p.

9. Daget, P. \& Poissonnet, J. (1971). Analyse phytologique des prairies. Critères d'application. Annale agronomique, 22 : 5-41.

10. Fenni, M. (2003). Étude des mauvaises herbes céréales d'hiver des hautes plaines constantinoises. Écologie, dynamique, phénologie et biologie des Bromes. Thèse de Doctorat Es. Sci., UFA Sétif, Maroc, $165 \mathrm{p}$.

11. Hatil, E., Manrayapin, J.P. \& Marnotte, P. (2008). Observation de l'enherbement en culture de canne à sucre en Guadeloupe. CTCS Guadeloupe. AFCAS, 13 p. 
12. Ipou Ipou, J., Adou, L.M.D., Touré, A., \& Marnotte, P. (2011). Aspects de la dynamique d'enherbement des parcelles par Euphorbia heterophylla L. (Euphorbiaceae) : production de graine et évolution du stock de semences. J. Appl. Biosci., 46: 3144- 3152.

13. Kouamé, D.K., Pene, C.B., Zouzou, M., Koulibaly, S.G., Tuo, K. \& Akpa, E.A. (2009). Evaluation agronomique de variétés de canne à sucre en début de campagne de récolte à Ferké au Nord de la côte d'ivoire : vers un allègement du schéma de sélection. Agronomie Africaine, 21 (3) : 319-330.

14. Lebreton, G. (2010). Effet de l'époque de coupe de la canne à sucre sur l'enherbement et le cycle biologique des adventices à la Réunion, Mémoire du Master, UFR Sciences de la Vie et de la Terre, Ecole Pratique des Hautes Etudes, France, 72 p.

15. Mangara, A., N'Da, A.A.A., Traoré, K., Kehe, M., Soro, K. \& Toure, M. (2010). Etude phytoécologique des adventices en cultures d'ananas (Ananas comosus (L.) Merr.) dans les localités de Bonoua et N'Douci en Basse Côte d'Ivoire. Journal of Applied Biosciences, 36 : 23672382.

16. Marion, D. \& Marnotte, P. (1991). Nuisibilité de l'enherbement sur une culture de canne à sucre. Rencontre internationale en langue française sur la canne à sucre, Nogent - sur- Marne, France, AFCAS, pp 188-191.

17. Marnotte, P. (2000). La gestion de l'enherbement et l'emploi des herbicides dans les systèmes de culture en zone Soudano sahélienne en Afrique de l'Ouest et du Centre. Formation du CIRAD. CIRAD-CAG.E.C.- AMATROP, 66 p.

18. Meddour, R. (2011). La méthode phytosociologique sigmatiste ou braun-blanqueto-tüxenienne. Faculté des Sciences Biologiques et Agronomiques, Département des Sciences Agronomiques, Tizi Ouzou, Algérie, 40 p.

19. Merlier, H. \& Montégut, J. (1982). Adventices tropicales. ORSTOM, CIRAD-GERDAT, ENSH, Montpellier, France, 490 p.

20. Pamentier, H. (2007). La carte de la vegetation de la Côte d'Ivoire. http:www.geoconfluences-ens-lsh.fr

21. Péné, B.C. \& Assa, A.D. (2003).Variations interannuelles de la pluviométrie et de l'alimentation hydrique de la canne à sucre en Côte d'Ivoire. Science et Changements Planétaire/Sécheresse. 14, 1, 43-52.

22. Péné, C.B. (1999). Gestion de l'irrigation en culture cannière : stratégies pour optimiser l'efficience d'utilisation de l'eau dans les périmètres sucriers ivoiriens. Doctorat d'État ès Sciences Naturelles, Université de Cocody, Côte d'Ivoire, 181 p. 
23. Raji, S. (1995). Concurrence des mauvaises herbes avec la culture de la canne à sucre (Saccharum officinarum L.) dans le Loukkos. Mémoire de 3ème cycle (Spécialité Productions Végétales), Institut Agronomique et Vétérinaire Hassan II, Rabat, Maroc, 95 p.

24. Sangaré, A., Koffi, E., Akamou, F. \& Fall, C.A. (2009). Rapport national sur l'état des ressources phytogénétiques pour l'alimentation et l'agriculture. In : Etat des ressources phytogénétiques pour l'alimentation et l'agriculture : Second rapport national, $65 \mathrm{p}$.

25. Sorensen, T. (1948). A method of establishing group of equal amplitude in plants sociology based on similary of species content. Det Kongelige danske videnkarbernes. Biologiske Skrifter, 5(4) : 1-34.

26. Traoré, K., Kouassi, K.C., Kouassi, R. H. \& Soro, D. (2014). Comparative study of the weed flora in Sugar Cane (Sacharum Officinarum L.) cultivation in the Area of the area of the Sugar refinery complex of Ferkessedougou. International Journal of Sciences research, 3 (1): 53-59.

27. Van Dillewijn, C. (1960). Botanique de la canne à sucre. Centre Technique de la Canne et du Sucre de la Guadeloupe et de la Martinique, $391 \mathrm{p}$. 